\title{
Modeling and Performance Analysis of Wi-Fi Networks Coexisting with LTE-U
}

\author{
Amr Abdelfattah and Naceur Malouch \\ Sorbonne Universités, UPMC Univ Paris 06, CNRS, LIP6 UMR 7606, 4 place Jussieu 75005 Paris \\ Email: \{amr.abdelfattah, naceur.malouch $\} @$ lip6.fr
}

\begin{abstract}
In order to cope with the exponential growth of mobile traffic, mobile operators need to access more spectrum resources. LTE in unlicensed spectrum (LTE-U) has been proposed to extend the usual operation of LTE in licensed spectrum to cover also unlicensed spectrum. However, this extension poses significant challenges especially regarding the coexistence between LTE-U and legacy systems like Wi-Fi. In case of LTE-U adopts Time-Division Multiplexing (TDM) schemes to share the spectrum with Wi-Fi, we expect performance degradations of Wi-Fi networks. In this paper, we quantify the impact of TDM schemes on Wi-Fi performance in a coexistence scenario. We provide detailed analytical models using two different random walk approaches to compute the probability of collision faced by Wi-Fi stations and their throughput performance. Besides, we derive the performance results using an exponential approximation which shows its insufficiency to capture the exact behavior. We implement the coexistence in the NS3 simulator and we show that the models estimate accurately the collision probability and the throughput experienced by Wi-Fi. The models are then used to study and compare different coexistence schemes showing for instance that the Wi-Fi frame size has a non-negligible impact on the performance of $\mathrm{Wi}-\mathrm{Fi}$ users.

Index Terms-LTE-U, CSAT, Wi-Fi, 5G, Mobile Communication, Collision Probability, Performance Evaluation, Simulation.
\end{abstract}

\section{INTRODUCTION}

Mobile operators pursue the increase the capacity of their cellular networks to cope with mobile data growth challenges [1]. As next generation communication systems (i.e. LTE, LTE-A) performance is close to Shannon bound in terms of spectrum efficiency, mobile operators move towards extending LTE operation in unlicensed spectrum (LTE-U), mainly at 5 $\mathrm{GHz}$ band due to its wide spectrum availability. However, this extension not only requires that LTE has to share the spectrum with other systems like Wi-Fi, but it requires also to share the spectrum fairly which is not part of its original design. Indeed, LTE commonly operates in licensed spectrum where the spectrum is exclusively reserved for its operation. This is precisely where the challenge comes through. Therefore, in coexistence scenarios, LTE-U has to adopt a new medium access control (MAC) protocol that should have two principle features: (i) It is a suitable MAC layer for LTE-U operation including uninterrupted and synchronous operation of the channel. (ii) It provides a fair coexistence with Wi-Fi. The key challenge is to simultaneously meet the above requirements which makes the design of a new LTE MAC layer quite intricate and remains open for further research. 3GPP proposes mainly two new MAC protocol categories for LTE [2]. The first is Listen Before Talk, LBT-based MAC protocol, that is LTE-U accesses the channel whenever it is sensed idle after waiting for a channel clear assessment period followed possibly by a backoff period. The second is TDM-based MAC protocol, called also Duty Cycled LTE or simply LTE-U, that is LTE-U accesses directly the channel without performing a clear channel assessment before. However, in a duty cycle, LTE-U must free the channel for some amount of time to offer transmission opportunities to Wi-Fi users.

In this paper, we investigate the fundamental question: How LTE-U can adopt efficiently a TDM-based protocol in order to coexist fairly with Wi-Fi? We answer this question through a sophisticated analysis that quantifies the negative impact of adopting TDM-based MAC protocol on Wi-Fi performance. We compute analytically the probability of collision between the two systems and we derive the Wi-Fi saturation throughput. Our contributions are:

- We combine several analytical models and refinements for a Wi-Fi network that operates alone over the channel based on a state-of-the-art review.

- We build on our refined model for Wi-Fi network to study the coexistence between LTE-U and Wi-Fi using two complementary analytical model approaches.

- We introduce the notion of random walk in studying 802.11 MAC performance. Our analytical analysis is general enough to be used in order to analyze other coexistence scenarios.

- We include the capture effect in the model to enable the consideration of classic and recent 802.11 PHY standards which introduce a number of novel features.

- We validate our analytical analysis through an extensive simulation study using NS3. We also develop a new module in NS3 simulator to simulate LTE-U coexisting with Wi-Fi network.

- We show that LTE-U negative impact on Wi-Fi performance could be compensated relying on our model. The impact of Wi-Fi packet size and TDM-based LTE-U parameters are highlighted.

The paper is outlined as follows. In Section II, we present related work. In section III, we provide the two analytical models for coexistence to quantify the impact of LTE-U on Wi-Fi performance. Section IV validates the accuracy of our models through NS3 simulations. There, we investigate the performance of Wi-Fi in various LTE-U TDM schemes and 
Wi-Fi configurations. Finally, Section V concludes the paper.

\section{RELATED WORK}

Several works have studied TDM-based (Duty Cycled) and LBT-based LTE-U in order to share the unlicensed spectrum with Wi-Fi networks.

The previous work [3] has studied the Duty Cycled LTE and Wi-Fi interaction through blank subframes in indoor environment for single and multiple-floor scenarios. Multiple TDM configurations have been examined using a proprietary semi-static system-level simulator. The results show first that the more the number of blank subframes over LTE frame, the higher the offered Wi-Fi throughput. Second, for the same number of blank subframes, Wi-Fi can experience different throughputs. These results were partially justified by the system-level simulator since the exact impact on the Wi-Fi performance was not analyzed. Thus, it is hard to compare between TDM schemes to find the best configuration parameters that maximize the throughput and reduce the collision probability. In this work, we provide a formal justification of the possible performance degradation experienced by WiFi. We show how the Wi-Fi performance is related to the number of blank subframes during the LTE frame and also to their distribution along the frame. Through the model and supported by NS3 simulations, we show that LTE-U duty cycle parameters and Wi-Fi packet size have an important role to increase or decrease the Wi-Fi throughput especially for low Wi-Fi channel bit rates. [4] has proposed CU-LTE, a cognitive coexistence general architecture based on clever channel selection to avoid interference with Wi-Fi. The proposed optimization framework can also determine how often the selected channels should be accessed, which corresponds to an important parameter for Duty Cycled LTE-U. In our work, we rather study details regarding frame collisions, IEEE 802.11 MAC protocol and all duty cycle parameters. In [5], TDM-based LTE-U has been examined to coexist with WiFi through simulations. Authors claimed that "LTE-U can be configured to be fair to Wi-Fi" without providing any further information about how to achieve it. Besides, they did not model the possible collisions between the two systems. Unlike [5] that approximates the Duty Cycled LTE-U scheme because of their proprietary simulator limitation, we integrate an exact Duty Cycled LTE-U in the well-known NS3 simulator. The probability of channel access for Wi-Fi stations is computed in [6] in presence of Duty Cycled LTE-U. It is based on the fact that during the LTE transmission period, Wi-Fi stations have to wait before accessing the channel, thus increasing the channel access. Neither the collision probability nor the Wi-Fi throughput can be derived using the computations in [6].

In a white paper published by Qualcomm [7], LBT-based LTE-U coexists with Wi-Fi in an outdoor simulation scenario, the simulation results show that LTE-U is a better neighbor to Wi-Fi than Wi-Fi to itself. However, [8] shows that when the load of LTE-U is very high, LBT impacts significantly the performance of Wi-Fi users while LTE-U users remain robust. This last result was based on the downlink performance of
Wi-Fi obtained via a Markov chain to model Wi-Fi random access to the spectrum. In [9], a modeling and analysis of LBT-based MAC protocol of LTE-U has been developed. It has been shown that a certain level of fairness with Wi-Fi can be achieved by adaptively adjusting the LBT parameters. Contrariwise to [9], our modeling of TDM-based LTE-U shows that we can achieve full fairness with Wi-Fi because our model is able to find the adequate configuration parameters. Besides, TDM-based LTE-U permits intrinsically to master the sharing between the two networks. The work in [10] shows through a system-level study that LBT-based LTE-U is more efficient to mitigate the interference among LTE-U base stations. For the interference to Wi-Fi, it is better to use dynamic channel selection than using LBT.

\section{WI-Fi/LTE-U ANALYTICAL Models}

Wi-Fi performance was studied through various analytical models that aim to capture the behavior and the procedures of the IEEE 802.11 MAC standard. Some of these models have successfully followed the standard in many technical aspects while they have failed to consider few other aspects. In the next subsection, based on a state-of-the-art review, we present the most recent and accurate analytical model that was established for a Wi-Fi network consisting of $n$ Wi-Fi stations. Indeed, we have harmonized between previous analytical models [11], [12], [13]. Then, we develop our novel analytical models for a Wi-Fi network that coexists with LTE-U based on two different approaches (subsection III-B).

\section{A. Model for Wi-Fi Networks}

First, as in [12], we use the same assumptions (i.e. saturation condition and ideal channel condition) to model the behavior of a single Wi-Fi station using a bidirectional discrete time Markov chain. Unfortunately, [12]'s Markov chain model and throughput analysis do not fit perfectly with the IEEE 802.11 MAC protocol. In [11], the authors proposed a first refinement by considering the finite retransmission limit of a Wi-Fi frame, which leads to a different Markov chain as shown in Figure 1. $W_{i}$ in the figure refers to the contention window (CW) of the

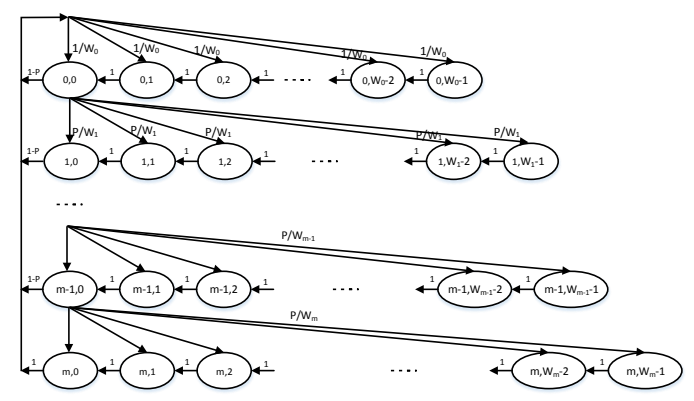

Fig. 1: Markov chain model for Wi-Fi stations.

$i^{\text {th }}$ backoff stage, $\mathrm{i} \in(0, \cdots, m)$ and $m$ is the maximum backoff stage or the maximum retransmission limit. The contention window $\mathrm{CW}$ is doubled whenever unsuccessful transmission 
occurs: $W_{i}=2^{i} W_{0}$, and $i \leq m^{\prime}$, where $m^{\prime}=\log _{2} \frac{C W_{\max }}{C W_{\min }}$ with $C W_{\min }=W_{0} . C W_{\max }$ denotes the minimum and maximum $\mathrm{CW}$ respectively. Whereas, for $i>m^{\prime}, \mathrm{CW}$ is hold on $C W_{\max }$.

The key parameter in the Markov chain of Figure 1 is $p$ which is the conditional collision probability, indicating that this is the probability of collision faced by a frame being transmitted on the channel. By considering that the Wi-Fi communication is divided into time slots and by solving the Markov chain for $m>m^{\prime}$, we determine the second key parameter $\tau$ which is the probability that a station transmits in a randomly chosen time slot. This is done by summing the probabilities of the states whenever the backoff counter equals zero, i.e. the states of the first column. This yields

$\tau=\frac{2\left(1-p^{m+1}\right)}{W_{0}\left(1-(2 p)^{m^{\prime}+1}\right) \frac{(1-p)}{(1-2 p)}+\left(1-p^{m^{\prime}+1}\right)+W_{0} 2^{m^{\prime}} p^{m^{\prime}+1}\left(1-p^{m-m^{\prime}}\right)}$

Besides, $p$ can be expressed as

$$
p=1-(1-\tau)^{n-1}
$$

Equations (1) and (2) establish a fixed point formulation from which $\tau$ and $p$ can be determined. These two parameters describe the transmission activity in the Wi-Fi network and are then used to compute the saturation throughput. In a randomly chosen slot, different situations can happen that define the state and the duration of the slot. First, if no station is transmitting during a slot, the state of slot is idle. Second, if exactly one station is transmitting, the slot experiences a successful transmission. Third, if more than one station transmits, the slot experiences a collision. Therefore, the slot is busy whenever at least one station transmits. In other words, a busy slot is a successful slot or a slot with collision.

Accordingly, let $\left(P_{i d}, T_{i d}\right),\left(P_{s}, T_{s}\right)$ and $\left(P_{c}, T_{c}\right)$ denote the pair of probability and duration of a slot in the states idle, successful and collision respectively. As in [13], it easily follows that

$$
\begin{aligned}
P_{i d} & =(1-\tau)^{n}, \quad T_{i d}=\delta \\
P_{s} & =n \tau(1-\tau)^{n-1}, \quad T_{s}=\left(T_{f}+D I F S\right)\left(\frac{W_{0}}{W_{0}-1}\right)+\delta \\
P_{c} & =1-P_{i d}-P_{s}, \quad T_{c}=T_{f}+D I F S+\delta
\end{aligned}
$$

Where $\delta$ is the empty slot time, DIFS is the Distributed InterFrame Space time and $T_{f}=T_{M P D U}+S I F S+T_{A C k}$ is the frame transmission time which includes the time to transmit the MAC Protocol Data Unit MPDU ( $\left.T_{M P D U}\right)$ followed by the Short Interframe Space time (SIFS) and the ACK transmission time $\left(T_{A C K}\right)$. Actually, the reason why $T_{s}$ is not equal to $T_{c}$ is due to the possibility of transmitting several successful frames by the same station in the same slot without interruption by other stations. Indeed, a station can win the access several times in a row. Such slot is called anomalous slot. This is another refinement of [12]'s model which has been explored in [13]. Now, we can express the normalized throughput $S$ as [13]

$$
\Gamma=\frac{P_{s} T_{P L}}{E[s l o t]}=\frac{P_{s} T_{P L}}{P_{i d} T_{i d}+P_{s} T_{s}+P_{c} T_{c}}
$$

Where $T_{P L}=\left(\frac{W_{0}}{W_{0}-1}\right) t_{P L}$ is the correction of $t_{P L}$, the packet payload duration, due to the anomalous slot. $E[$ slot $]$ denotes the mean slot duration.

\section{B. Wi-Fi coexists with LTE-U}

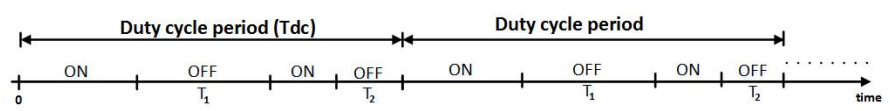

Fig. 2: Duty Cycled LTE (LTE-U)

Now we consider the coexistence scenario where LTE-U shares the spectrum with $n$ Wi-Fi stations using a TimeDivision Multiplexing (TDM) scheme. As shown in Figure 2, LTE-U transmission is activated/deactivated according to an on/off pattern to form the so-called Duty Cycled LTE which is configured by different parameters. The duty cycle period $T_{d c}$ that determines how long it takes to the pattern to be repeated again. The number of off-periods $K$ over the duty cycle period which represents the number of disjoint access channel opportunities offered to Wi-Fi during a duty cycle period. Finally, the duty cycle percentage which is the portion of time where the LTE-U is activated over the duty cycle period. Practically speaking, Wi-Fi access the channel during different durations of LTE-U off-periods over each duty cycle. Let us adopt the notation $T_{k}$ for such off-periods where $\mathrm{k} \in(1, \cdots, K)$. The tying up between Duty Cycled LTE configuration parameters (i.e. $K, T_{k}$ and $T_{d c}$ ) and Wi-Fi performance is one of the important theoretical contributions of this work. As a result, it becomes malleable to configure LTE-U parameters so that the spectrum is shared adequately between the two systems.

Evidently, a first LTE-U negative impact on Wi-Fi performance can be captured by a positive drift in the collision probability $p$ shown in Figure 1 and computed in equation (2). It is clear that this drift is considered only when an LTE-U onperiod starts during an ongoing Wi-Fi successful transmission, or by another meaning, an ongoing Wi-Fi transmission without collisions with other Wi-Fi transmissions. As a matter of fact, when LTE-U on-period starts during a collision among Wi-Fi stations, it does not cause an additional collision. Nevertheless, we will see later that the starting time of the LTE-U onperiod does impact the Wi-Fi performance even during a WiFi collision. We define the LTE-U probability, $p_{l t e}$, to be the probability that an on-period starts during an ongoing transmission whether it is a successful transmission or not. Thus, the new collision probability of Wi-Fi coexisting with LTE-U can be rewritten as

$$
p=1-(1-\tau)^{n-1}+(1-\tau)^{n-1} p_{\text {lte }}
$$

Our strategy is best understood by remarking that the "interaction" between the two networks occurs exactly at the end of each $T_{k}$ period, possibly in the form of a collision. Therefore, the first step of our strategy is to find an appropriate expression for $p_{\text {lte }}$ to take into account the impact of LTE-U on Wi-Fi. Accordingly, equations (1) and (5) formulate our new 
fixed point system which will enable us to determine $\mathrm{Wi}-\mathrm{Fi}$ throughput. To do so, we provide two different approaches based on how we model the transmissions in the Wi-Fi network during LTE-U off-periods. In the second step of our strategy, we provide a new $\mathrm{Wi}-\mathrm{Fi}$ throughput formulae different than equation (4) depending on the adopted modeling approach. We call the two approaches as slot-by-slot random walk and frame-by-frame random walk.

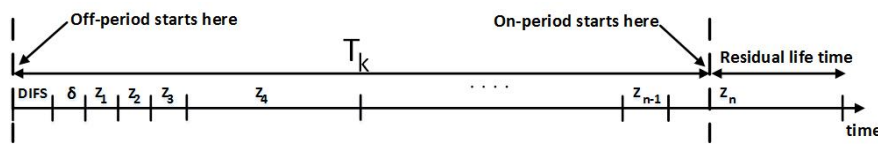

Fig. 3: Slot-by-Slot random walk model for Wi-Fi.

1) Slot-by-Slot Random Walk: In this approach, in order to build our new fixed point system and determine $p_{\text {lte }}$, we model Wi-Fi transmissions during LTE-U off-periods as a random walk interrupted or stopped by LTE-U arrival, i.e. starting of LTE-U on-period (Figure 3). The random variable that defines the random walk corresponds to the slot duration. Remember a slot can be empty or busy as mentioned in the previous subsection. The slot-by-slot random walk describes the fact that Wi-Fi transmissions are slotted and thus constitutes a succession of slots alternating randomly between idle and busy. Notice that at the beginning of each off-period, WiFi stations have to wait for a period of DIFS $\delta$ before starting any transmissions [14]. Thus, the random walk starts at $D I F S+\delta$ and terminates at $T_{k}$. Let $Z$ be the random variable representing the slot duration. Its probability density function is the following

$$
P(Z=T)=\left\{\begin{array}{lll}
P_{b} & \text { for } & T=T_{b} \\
P_{\text {id }} & \text { for } & T=T_{i d}
\end{array}\right.
$$

where $P_{b}=P_{s}+P_{c}$ denotes the probability that the slot is a busy slot and $T_{b}=T_{f}+D I F S+\delta$ is its duration. Only at this stage of our analysis we forsake the second refinement of [12]'s model (i.e. $T_{s} \neq T_{c}$ ) in our random walk since the second refinement provides the average of the anomalous slot while here we need full slots. Thus, two successive successful transmissions are fully counted in the random walk. Now, let $N_{k}$ be the random variable that counts the number of slots which could be occurred during any $T_{k}$ period. A slot that has been stopped by an on-period without terminating is considered as it has been occurred (See Figure 3 ). Consequently, the probability that $n$ slots occur during any $T_{k}$ period can be written as [15]

$$
\begin{array}{r}
\operatorname{Pr}\left\{N_{k}=n\right\}=\operatorname{Pr}\left\{\sum_{j=1}^{n-1} z_{j}<T_{k}-\text { DIFS }-\delta\right\} \\
-\operatorname{Pr}\left\{\sum_{j=1}^{n} z_{j}<T_{k}-\text { DIF } S-\delta\right\}
\end{array}
$$

For a given $T_{k}$ period, $N_{k}$ has an upper bound $\max$, which means that $\operatorname{Pr}\left\{N_{k}>n \max \right\}=0$, where $\operatorname{nmax}=\left\lceil T_{k} / \delta\right\rceil$. Hence, we compute the expected number of busy slots that occur in $T_{k}$ period, $E\left[N_{k}^{b}\right]$, as follows

$$
E\left[N_{k}^{b}\right]=P_{b} E\left[N_{k}\right]=P_{b} \sum_{n=1}^{n \max } n \operatorname{Pr}\left\{N_{k}=n\right\}
$$

According to $p_{\text {lte }}$ definition mentioned above, the collision occurs only if the last slot is a busy slot and the on-period starts exactly during the frame transmission portion of the busy slot which means $T_{f}$ in equation (3). Unfortunately, the slot-byslot model is limited because it does not dissociate between the two portions of time inside a busy slot which are $T_{f}$ and $D I F S+\delta$. This is due to the slot vision defined in [12] and that we are using in this first approach. It leads to not knowing when exactly the on-period starts during a busy slot. We can still approximate $p_{\text {lte }}$ as follows

$$
p_{\text {lte }}=\frac{K}{\sum_{k=1}^{K} E\left[N_{k}^{b}\right]}
$$

The numerator of the above formula is an approximation of the average number of busy slots that occur at the end of the off-period (random walk). In other words, it is the average number of off-periods terminating with a busy slot. Typically, this number is lower or equal to $K$. The approximation relies on the fact that the total duration of idle slots is very low compared to the duration of frame transmissions, so that the probability of having an on-period starts at an idle slot is low.

Now, similarly to equation (4), we express the Wi-Fi throughput over a duty cycle period as follows

$$
\Gamma_{1}=\frac{\left(P_{s}\left(1-p_{\text {lte }}\right) \sum_{k=1}^{K} E\left[N_{k}\right]\right) * T_{P L}}{T_{d c}}
$$

where $P_{s}\left(1-p_{\text {lte }}\right)$ is the probability that a slot is successful and the transmitted frame in that slot does not collide with LTE-U. We can show that $N_{k}$ is a stopping time with respect to $\left\{z_{j}\right\}$ because $\operatorname{Pr}\left\{N_{k}=n\right\}$ is totally determined through all $z_{j}, j \in 1 \cdots n$. Hence, using Wald's equality [15], we have

$$
E\left[N_{k}\right] E[Z]=T_{k}-D I F S-\delta+E\left[R_{k}\right]
$$

where $E\left[R_{k}\right]$ represents the expected residual life time of $\sum_{j=1}^{n} z_{j}, n \geq 1$ with respect to $T_{k}-D I F S-\delta$. Actually, $E\left[R_{k}\right]$ brings the second and more important information required to adjust the throughput in (4) to take into account the coexistence with LTE-U. This becomes clear by remarking that $E[Z]=E[$ slot $]$ and by substituting equation (11) into equation (10). We obtain the modified version of (4) which takes into account the coexistence with LTE-U.

$$
\Gamma_{1}=\Gamma *\left(1-p_{\text {lte }}\right) \frac{\sum_{k=1}^{K}\left(T_{k}-D I F S-\delta+E\left[R_{k}\right]\right)}{T_{d c}}
$$

To summarize, in order to compute the Wi-Fi throughput coexisting with LTE-U through equation (12), we determine first $p$ and $\tau$ using the new fixed point system which involves equation (9) as well. Then, we compute $p_{\text {lte }}$ using (9) and $E\left[R_{k}\right]$ using equations (7), (8) and (11) (Wald's equality). It is worthwhile noticing that the formula of throughput in (12) 
is general enough so that any other method or approximation can be used to compute $p_{\text {lte }}$ and $E\left[R_{k}\right]$. Hence, naturally we show in the next paragraph how to compute the coexistence throughput using the classic exponential approximation. Also, other LTE-U on/off patterns can be studied using the model because they are captured by the two parameters $p_{\text {lte }}$ and $E\left[R_{k}\right]$

Exponential Model for LTE-U. Here we assume that the LTE-U off-period time is drawn from an exponential distribution with a mean equals to the real fixed duration of the off-period.

Let's first compute $p_{\text {lte }}$ for an exponential off-period with mean $T_{k}$. It is equal to the probability that the frame transmission time $T_{f}$ is larger than the remaining time of the off-period, formally written exactly as follows

$$
\operatorname{Pr}\left\{\text { off-period } \leq T_{f}\right\}=1-e^{T_{f} / T_{k}}
$$

This is because the distribution of the remaining time is also exponentially distributed with the same mean. Equivalently, we can see LTE-U arrivals as a Poisson process with mean $\Re_{k}=\frac{1}{T_{k}}$. For $K$ off-periods, we can write approximately

$$
p_{\text {lte }}=1-e^{\Re T_{f}}, \Re=K /\left(\sum_{k=1}^{K} T_{k}\right)
$$

where $\Re$ is LTE-U arrival rate during all off-periods. Alternatively, we can compute the probability as a weighted sum by conditioning on the probability that a frame is being transmitted in the $k^{t h}$ off-period:

$$
p_{\text {lte }}=\sum_{k=1}^{K} T_{k}\left(1-e^{\Re_{k} T_{f}}\right) / \sum_{k=1}^{K} T_{k}
$$

Our simulation results show that both approximations are acceptable. Solving (14) (or (15)), (5) and (1) yields $\tau$. To calculate the throughput in (12), we determine $E\left[R_{k}\right]$ as

$$
E\left[R_{k}\right]=P_{b}\left(T_{b}-E\left[T T_{b}\right]\right)+P_{i d}(\delta-E[T \delta])
$$

where $E\left[T T_{b}\right]$ is the expected time before on-period starts during a $T_{b}$ period. $E[T \delta]$ is defined equivalently. These are calculated using standard formulae for truncated exponential distributions:

$$
E\left[T T_{b}\right]=\frac{1}{\Re_{k}}-\frac{T_{b} e^{-\Re_{k} T_{b}}}{1-e^{-\Re_{k} T_{b}}}, E[T \delta]=\frac{1}{\Re_{k}}-\frac{\delta e^{-\Re_{k} \delta}}{1-e^{-\Re_{k} \delta}}
$$

2) Frame-by-Frame Random Walk: To avoid the approximation made in (9), we model the Wi-Fi transmission during off-periods as a succession of transmission rounds stopped by LTE-U arrivals. This forms our frame-by-frame random walk because in each round there will be only one $T_{f}$ period (Figure 4). Remember during a $T_{f}$ period, we can have one or several transmitted frames in parallel. A transmission round consists of a random backoff period, followed by the frame transmission(s) period $T_{f}$, terminated by a sensing period $D I F S$. Let $X$ be the random variable denoting the amount of time needed to each transmission round

$$
X=\delta * B F+T_{f}+D I F S
$$

Where $B F$ is a random variable that depicts the backoff counter before the frame transmission(s) period. To fully determine $X$, we define the probability mass function of $B F$ as

$$
P(B F=j)=\left\{\begin{array}{lll}
\frac{1}{\eta W_{0}} & \text { for } & j=0 \\
\frac{1}{\eta}\left(1-P_{b}\right)^{j} P_{b} & \text { for } & 1 \leq j \leq W_{m-1}
\end{array}\right.
$$

Where

$$
\eta=\left(1 / W_{0}\right)+\left(1-P_{b}\right)\left(1-\left(1-P_{b}\right)^{W_{m}-1}\right)
$$

is a normalization term to ensure that the probability of each backoff counter follows a valid probability distribution. To clarify, the $B F$ 's distribution cannot follow a geometric distribution because of the existence of the anomalous slots mentioned in [13]. Now, let $M_{k}$ be the random variable that counts the number of rounds occurred during any $T_{k}$ period. According to (18), the total time needed to have $m$ rounds during any $T_{k}$ period is equal to $\sum_{j=1}^{m} x_{j}$. Hence, the probability of having $m$ rounds during any $T_{k}$ period can be written as

$$
\begin{aligned}
\operatorname{Pr}\left\{M_{k}=m\right\} & =\operatorname{Pr}\left\{\sum_{j=1}^{m-1} x_{j}<T_{k}-D I F S-\delta\right\} \\
& -\operatorname{Pr}\left\{\sum_{j=1}^{m} x_{j}<T_{k}-D I F S-\delta\right\}
\end{aligned}
$$

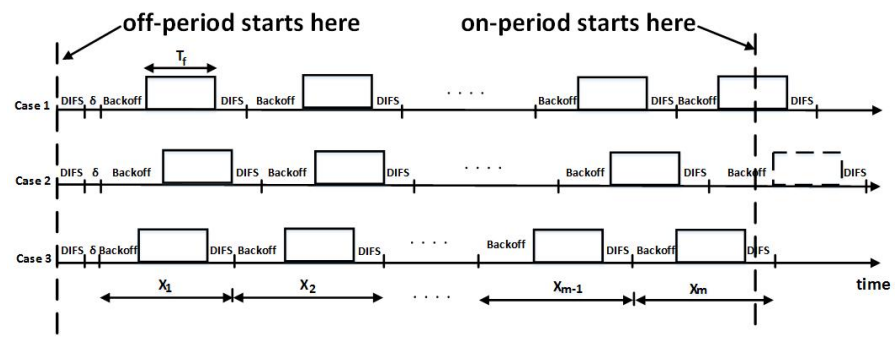

Fig. 4: Frame-by-Frame random walk model for Wi-Fi

We also emphasize that the above probability is the probability that the first $m-1$ rounds were fully terminated without interruption by the next on-period whereas the $m^{t h}$ round is interrupted by the starting of the next on-period. This interruption happens according to three possible scenarios. (i) on-period starts during $T_{f}$, (ii) on-period starts during DIFS, and (iii) on-period starts during backoff period. We call the corresponding rounds collided, successful and frozen respectively. We compute the probability of each scenario for a given $m$ as follows

$$
\begin{aligned}
& \operatorname{Pr}\left\{c_{k}, m\right\}=\operatorname{Pr}\left\{\sum_{j=1}^{m} x_{j}-T_{f} \leq T_{k} \leq \sum_{j=1}^{m} x_{j}\right\} \\
& \operatorname{Pr}\left\{s_{k}, m\right\}=\operatorname{Pr}\left\{\sum_{j=1}^{m} x_{j}<T_{k} \leq \sum_{j=1}^{m} x_{j}+D I F S+\delta\right\} \\
& \operatorname{Pr}\left\{f_{k}, m\right\}=\operatorname{Pr}\left\{\sum_{j=1}^{m-1} x_{j}+D I F S+\delta<T_{k}<\sum_{j=1}^{m} x_{j}-T_{f}\right\}
\end{aligned}
$$


For a given $T_{k}$ period, $M_{k}$ has an upper bound $\operatorname{mmax}$, where $\operatorname{mmax}=\left\lceil T_{k} / x \min \right\rceil$ with $x \min =D I F S+T_{f}$. Besides, we notice that the event $\left\{M_{k}=m\right\}$ is included in the event $\left\{c_{k}, m\right\}$. Hence, the probability that the last round in an offperiod is a collided one is expressed as

$$
\operatorname{Pr}\left\{c_{k}\right\}=\sum_{m=1}^{\operatorname{mmax}} \operatorname{Pr}\left\{c_{k}, m\right\}
$$

$\operatorname{Pr}\left\{s_{k}\right\}$ and $\operatorname{Pr}\left\{f_{k}\right\}$ are similarly written as (22) from (21). Now, $p_{l t e}$ is calculated over a duty cycle period as

$$
p_{l t e}=\frac{\sum_{k=1}^{K} \operatorname{Pr}\left\{c_{k}\right\}}{\sum_{k=1}^{K}\left(E\left[M_{k}\right]-\operatorname{Pr}\left\{f_{k}\right\}\right)}
$$

where $E\left[M_{k}\right]=\sum_{m=1}^{\operatorname{mmax}} m \operatorname{Pr}\left\{M_{k}=m\right\}$ is the expected number of transmission rounds during a $T_{k}$ period regardless of the $m^{t h}$ round was a collision, successful or frozen round. The $\operatorname{Pr}\left\{f_{k}\right\}$ term is necessary to not count the frozen round.

Up to this point of the analysis, equations (23), (5) and (1) represent our new fixed point formulation. Now, we compute differently the throughput based on counting the number of successful Wi-Fi frames during off-periods unlike (12). In fact, during $T_{k}$ period, the expected number of successful rounds, $E\left[S_{k}\right]$, can be calculated by using the fact that the first $m-1$ rounds are of type successful, which means no interruption by LTE-U, whereas the last one is only successful with probability $\operatorname{Pr}\left\{s_{k}\right\}$. Hence,

$$
E\left[S_{k}\right]=E\left[M_{k}\right]-1+\operatorname{Pr}\left\{s_{k}\right\}
$$

For the throughput, we consider only the ratio of $E\left[S_{k}\right]$ where only one Wi-Fi station transmits alone on the channel. Simply, the ratio is the probability that a slot is a successful slot given it is a busy slot denoted by $P_{s \mid b}=P_{s} / P_{b}$. Finally, the Wi-Fi throughput is expressed as

$$
\Gamma_{2}=\frac{P_{s \mid b} \sum_{k=1}^{K} E\left[S_{k}\right]}{T_{d c}} \quad \text { frame } / \mathrm{sec}
$$

\section{Accounting for the capture effect}

So far, the analytical models have focused on capturing the behavior of the MAC layer. It is possible though to consider the capture effect [16] between Wi-Fi and LTE-U that is a WiFi frame can survive the collision with LTE-U transmission and be successfully received. It leads also to account for several PHY layer parameters such as transmission powers and antenna gains. The capture probability between LTE-U and $\mathrm{Wi}-\mathrm{Fi}$ is equal to $(1-B E R)^{L}$ where $L$ is the number of noisy bits [17]. Equivalently, the capture probability is equal to $(1-B E R)^{C B R \times r}$ where $r$ is the overlapping duration between the LTE-U and Wi-Fi transmission. Thus, the capture probability is computed as

$$
p_{\text {cap }}=\sum_{k=1}^{K} \int_{0}^{T_{b}} p d f_{R_{k}}(r)(1-B E R)^{C B R \times r} \mathrm{~d} r / K
$$

where $p d f_{R_{k}}()$ is the probability distribution function of the residual life time for the $k^{t h}$ off-period. CBR is the Wi-Fi Channel Bit Rate and BER is the bit error rate probability calculated depending on the signal to noise and interference ratio (SINR) and the modulation and coding scheme. In general, it is not tractable to express the distribution of $R_{k}$. We can use the Jensen's Inequality to approximate $p_{c a p}$ as follows

$$
p_{\text {cap }} \approx \frac{\sum_{k=1}^{K}(1-B E R)^{C B R \times E\left[R_{k}\right]}}{K}
$$

In reality, the above expression is a lower bound on the capture probability because $(1-B E R)^{C B R \times r}$ is a convex function. Accordingly, (5) must be modified to take into consideration the capture effect as

$$
p=1-(1-\tau)^{n-1}+(1-\tau)^{n-1} p_{\text {lte }}\left(1-p_{\text {cap }}\right)
$$

Now, (28) and (1) formulate our new fixed point system that yields $\tau$. Next, we similarly revise the throughput formula (12) to account for the capture effect as follows

$$
\Gamma_{3}=\Gamma * \frac{\left(1-p_{l t e}\left(1-p_{c a p}\right)\right) \sum_{k=1}^{K}\left(T_{k}-D I F S+E\left[R_{k}\right]\right)}{T_{d c}}
$$

\section{MODEL VALIDATION AND RESULTS}

To validate our analytical model, we simulate different coexistence scenario between $\mathrm{Wi}-\mathrm{Fi}$ and LTE-U using NS3 simulator. We set up a IEEE 802.11 network consisting of $n$ stations with configuration parameters as reported in Table I. We implemented a new module in NS3 simulator to simulate LTE-U transmissions. The LTE-U transmission duty cycle period is fixed to $10 \mathrm{~ms}$ corresponding to LTE frame duration with duty cycle percentage of $50 \%$. We adopt different configurations of the duty cycle as follows. $5 \mathrm{x} 0$ configuration: LTE-U transmission is activated for $5 \mathrm{~ms}$ then deactivated for the rest of LTE frame duration. 3x2 configuration: LTE-U transmission is activated for $3 \mathrm{~ms}$ then deactivated for same amount of time before it is reactivated again for $2 \mathrm{~ms}$. Consequently, the Wi-Fi gets two disjoint transmission opportunities in the same LTE frame to access the channel $(K=2)$. Finally, $4 \times 1$ configuration: similar to the previous one except we replace $3 \mathrm{~ms}$ by $4 \mathrm{~ms}$, and we replace $2 \mathrm{~ms}$ by $1 \mathrm{~ms}$.

\section{A. Validation Through Simulation and Observations}

We first verify the accuracy and the utility of our refined model of Wi-Fi when it operates alone on the channel. Figures 5 and 6 show that our refined model is the closest to simulation results for both collision probability $(p)$ and throughput $(\Gamma)$ in comparison with the other analytical models.

Now, we turn our attention to the coexistence between WiFi and LTE-U. We start by validating our final frame-byframe random walk model against NS3 results as shown in Figures 7 and 8 for a 12Mbps channel. To clarify the impact of LTE-U on Wi-Fi, we plot also in the same Figures the Wi-Fi collision probability and throughput performance when Wi-Fi operates alone on a $6 \mathrm{Mbps}$ channel. This is referred as $W i-F i$ on the Figures. In coexistence scenario, the model results follow exactly the behavior of simulations for both collision probability and throughput $\left(\Gamma_{2}\right)$. The relative error 


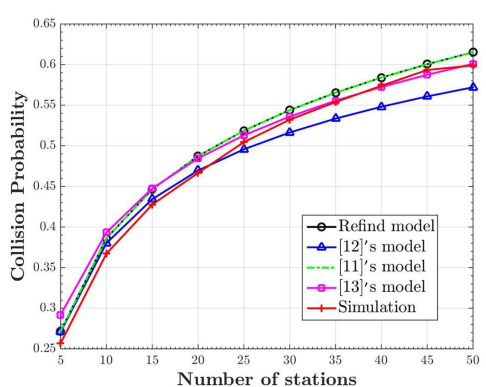

Fig. 5: Collision probability versus network size: pksize $=1500$ bytes and $\mathrm{CBR}=6 \mathrm{Mbps}$.

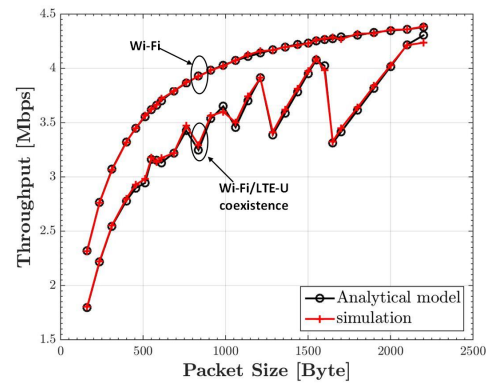

Fig. 8: Frame-by-Frame model. Throughput versus packet size: $\mathrm{n}=10, \mathrm{CBR}=$ 12 Mbps and $5 \times 0$ config.

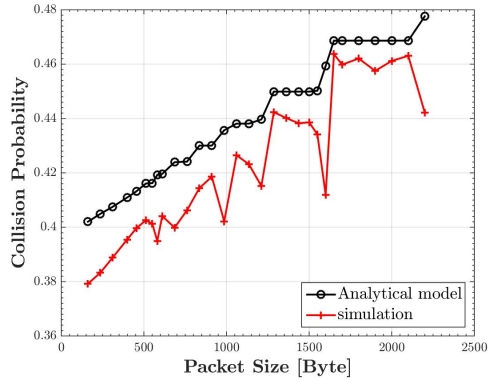

Fig. 11: Slot-by-Slot model. Collision probability versus packet size: $\mathrm{n}=10, \mathrm{CBR}=12$ Mbps and 5x0 config.

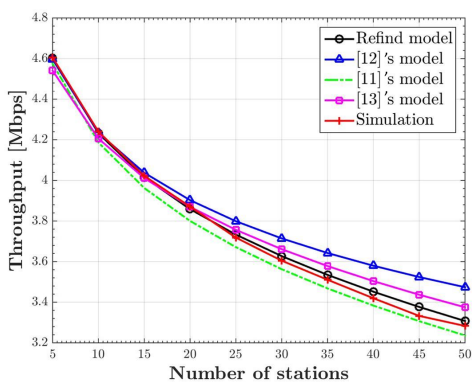

Fig. 6: Throughput versus network size: pksize $=1500$ bytes and CBR $=6$ Mbps.

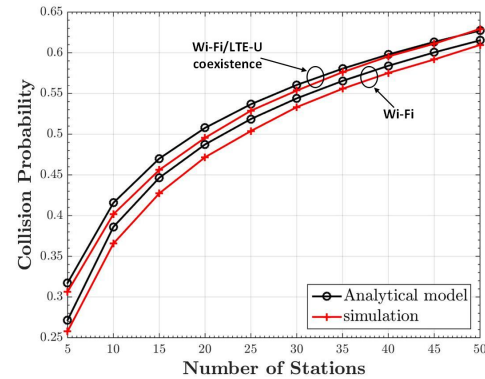

Fig. 9: Frame-by-Frame model. Collision probability versus network size: pksize $=512$ bytes, $\mathrm{CBR}=12 \mathrm{Mbps}$ and $5 \times 0$ config.

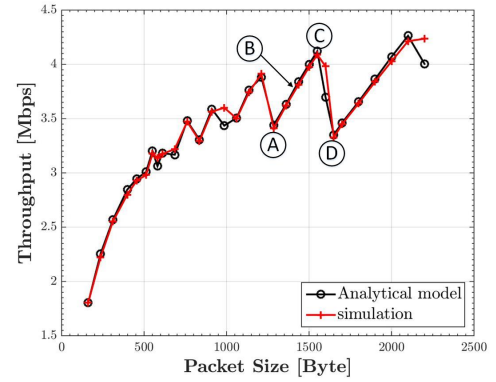

Fig. 12: Slot-by-Slot model. Throughput versus packet size: $\mathrm{n}=10, \mathrm{CBR}=12 \mathrm{Mbps}$ and $5 \times 0$ config.

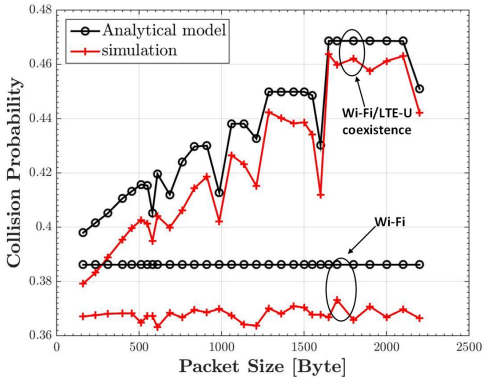

Fig. 7: Frame-by-Frame model. Collision probability versus packet size: $\mathrm{n}=10, \mathrm{CBR}$ $=12 \mathrm{Mbps}$ and $5 \mathrm{x} 0$ config.

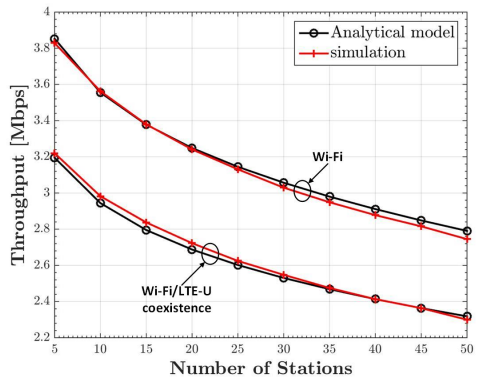

Fig. 10: Frame-by-Frame model. Throughput versus network size: pksize $=512$ bytes, $\mathrm{CBR}=12 \mathrm{Mbps}$ and $5 \mathrm{x} 0$ config.

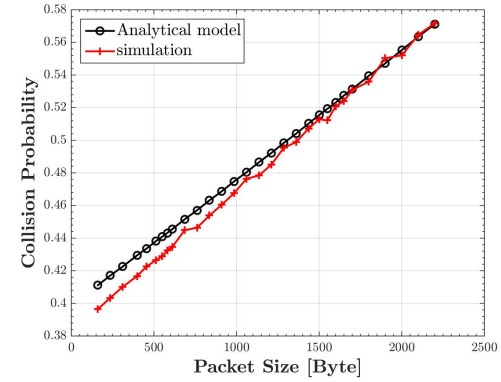

Fig. 13: Exponential model. Collision probability versus packet size: $\mathrm{n}=10, \mathrm{CBR}=12$ Mbps and $\Re=400 s^{-1}$.

\begin{tabular}{lr|lr}
\hline Simulation time & $50 \mathrm{~s}$ & MAC header & 36 bytes \\
Packet size (pksize) & $11 \sim 2200$ bytes & CBR & 6,12 and 24 Mbps \\
Channel bandwidth & $20 \mathrm{MHz}$ & Slot time $(\delta)$ & $9 \mu \mathrm{s}$ \\
ACK packet & 14 bytes & SIFS & $16 \mu \mathrm{s}$ \\
UDP header & 8 bytes & DIFS & $34 \mu \mathrm{s}$ \\
IP header & 20 bytes & & \\
\hline
\end{tabular}

TABLE I: Wi-Fi configuration parameters used in the comparison between NS3 simulations and the analytical model

observed from the model of Wi-Fi alone is kept the same for the coexistence meaning that our frame-by-frame random walk model is able to find precisely the additional impact of LTE-U on Wi-Fi. We will analyze and explain the plots later on.

Moreover, we continue to validate our model for different numbers of Wi-Fi stations as shown in Figures 9 and 10.

Figures 11 and 12 present the analytical results obtained by the slot-by-slot random walk model. We observe that globally the model is very accurate except for certain packet sizes, e.g. 986 and 1600 bytes where the model overestimates the collision probability. This is attributed to the approximation made in equation (9). Actually, the overestimation happens when the probability that the on-period starts during a DIFS or backoff period is high.

Since we have shown the utility of the slot-by-slot model to accommodate another LTE-U traffic model such as exponential LTE-U off-periods, we plot the corresponding results in Figures 13 and 14. These plots emphasize on the fact that duty cycled LTE are not well approximated by an exponential LTE-U because it is not able to capture the ripples seen in previous Figures 7, 8, 11 and 12 .

\section{B. Wi-Fi/LTE Coexistence Model Analysis and Application}

Let us first analyze probably the most eye-catching observation regarding the throughput ripples which becomes even 


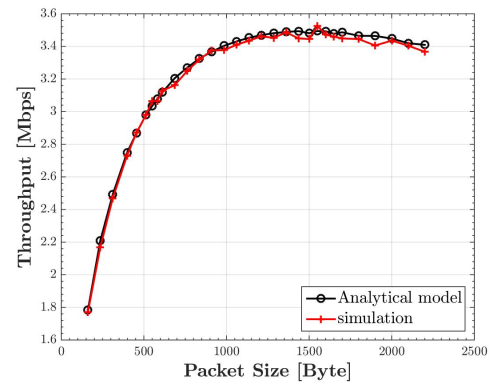

Fig. 14: Exponential model. Throughput versus packet size: $\mathrm{n}=10, \mathrm{CBR}=12 \mathrm{Mbps}$ and $\Re=400 s^{-1}$.

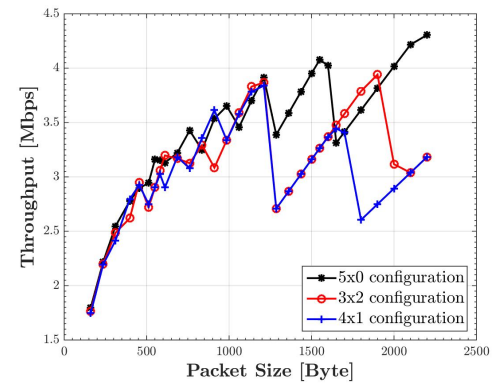

Fig. 15: Frame-by-Frame model. Comparison of the three configurations: $\mathrm{n}=10$ and $\mathrm{CBR}=12 \mathrm{Mbps}$.

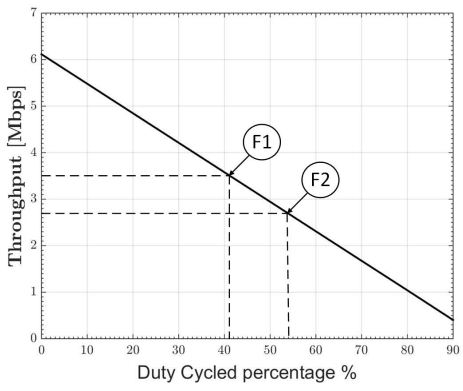

Fig. 16: Frame-by-Frame model. LTE-U duty cycle percentage fairness operation points: $\mathrm{n}=10$, pksize $=512$ bytes, $\mathrm{CBR}=12 \mathrm{Mbps}$ and $\mathrm{K}=1$.

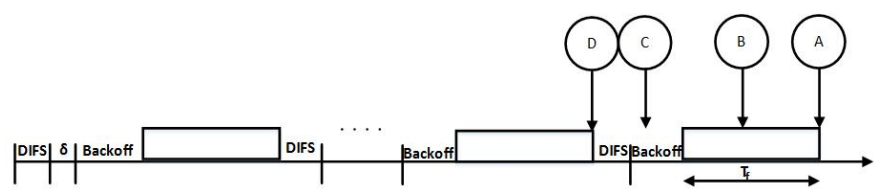

Fig. 17: LTE-U arrival possibilities during off-period

sharper for large packet sizes in Figures 8 and 12. Particularly, in Figure 12, we highlight four points and we analyze them through Figure 17. At point A, the LTE-U transmission starts most often at the tail of Wi-Fi frames which means the end of $T_{f}$ period, leading to a maximum damage on Wi-Fi throughput. This is because the whole time spent for $\mathrm{Wi}-\mathrm{Fi}$ transmission(s) is wasted in extremis. In this case, the expected residual life time $E\left[R_{k}\right]$ is close to zero and thus the numerator in the throughput equation (12) is minimized.

From A to B, as the packet size increases the time spent for Wi-Fi transmission(s) before LTE-U interruption decreases. In this situation, LTE-U starts during $T_{f}$ period, which increases the residual life time of Wi-Fi transmission(s) which in turn increases linearly the throughput. In addition, the throughput increases due to the reduction in the overhead induced by headers. This explains the following result which is at first glance counter-intuitive: The performance when LTE-U starts at the beginning of the frame transmission(s) is better than the performance when LTE- $U$ starts at the end.

From $\mathrm{B}$ to $\mathrm{C}$, the increase of the packet size switches the LTE-U arrival over the backoff period or the DIFS period which corresponds evidently to a maximization of the WiFi throughput and a minimization of the collision probability. Finally, from $\mathrm{C}$ to $\mathrm{D}$, we move sharply to another minimum point mainly due to the fact that the number of frames that can be sent during the off-period is reduced abruptly by one.

This rippling behavior is reflected on the collision probability as well, Figures 7 and 11. Indeed, it starts by a positive linear relation over a small range of small packet sizes. Then, it shows some stairs separated by fissures that increase in magnitude as the packet size increases.

1) Comparison Between Different LTE-U Configurations: Using our analytical models, we compare between different duty cycled LTE coexistence configurations with Wi-Fi. Figure
15 demonstrates that $5 \times 0$ configuration is the best configuration to coexist with Wi-Fi where the throughput is globally the highest for the most of packet sizes. This is justified by the fact that $3 \times 2$ and $4 \times 1$ configurations approximately double the collision probability between LTE-U and Wi-Fi relatively to the one obtained with $5 \times 0$ configuration. On the other hand, $4 \times 1$ configuration shows lower throughput than $3 \times 2$ configuration because $1 \mathrm{~ms}$ is not long enough to allow Wi-Fi transmission without frequent collisions with LTE-U.

2) Controlling Wi-Fi/LTE Coexistence Using the Model: Relying on our analytical model, an effective solution to compensate the negative impact of LTE-U on Wi-Fi is to modify the duty cycle percentage so that we master better the sharing between the two networks. Figure 16 represents a straightforward solution by modifying the duty cycle percentage of LTE-U. Point F1 in Figure 16 corresponds to the LTE-U duty cycle percentage that should be configured to allow WiFi throughput to achieve the equivalent throughput as if the Wi-Fi network operates alone on the channel with a bit rate equals half the coexistence channel $(6 \mathrm{Mbps}=12 \mathrm{Mbps} / 2$, is used in the Figure). We can consider indeed in this situation as if the channel has been equally shared by Wi-Fi and LTE$\mathrm{U}$. To do so, the duty cycle percentage is reduced here from $50 \%$ to $41 \%$. The difference of $9 \%$ can be considered as a necessary overhead for LTE-U to use the Wi-Fi channel.

Another sharing point of view consists for LTE-U to consume the same bandwidth that would be consumed by another Wi-Fi network having the same properties as the target WiFi network. In other words, if the Wi-Fi network includes 10 stations, then their bandwidth share should be the same to what they would get if the network was 20 stations. So it is like LTE-U is behaving as $10 \mathrm{Wi}-\mathrm{Fi}$ stations (to be Wi-Fifriendly). This definition of fairness was mentioned by 3GPP in [2]. The point F2 in Figure 16 corresponds to the LTE duty cycle percentage to achieve this type of fairness. In this case, the percentage increases from $50 \%$ to $55 \%$. It means that it is possible to configure a percentage larger than $50 \%$ for LTE-U and still be fair with Wi-Fi. It means also that a percentage of $50 \%$ provides to the Wi-Fi network better performance than another similar Wi-Fi network!

Another way to improve the Wi-Fi throughput is by increas- 


\begin{tabular}{|c|l|l|l|l|l|l|l|l|l|l|l|}
\hline SIR [dB] & 0 & 1 & 2 & 3 & 4 & 5 & 6 & 7 & 8 & 9 & 10 \\
\hline Analytical & 3.01 & 3.01 & 3.01 & 3.01 & 3.03 & 3.13 & 3.21 & 3.24 & 3.24 & 3.24 & 3.24 \\
\hline Simulation & 2.98 & 2.98 & 2.98 & 2.98 & 2.98 & 2.98 & 3.1 & 3.21 & 3.2 & 3.2 & 3.21 \\
\hline
\end{tabular}

TABLE II: Slot-by-Slot model. Throughput versus SIR: $\mathrm{n}=10$, pksize $=512$ bytes, $\mathrm{CBR}=12 \mathrm{Mbps}$ and $5 \mathrm{X} 0$ config.

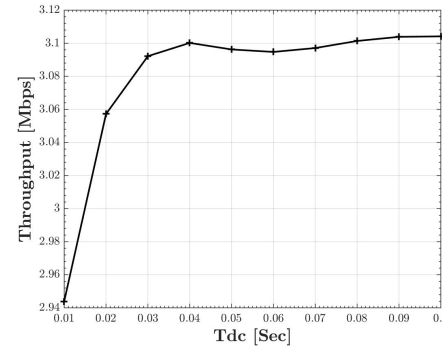

Fig. 18: Throughput versus duty cycle period: $\mathrm{n}=10$, pksize $=512$ bytes, $\mathrm{CBR}=12 \mathrm{Mbps}, 5 \mathrm{X} 0$ config.

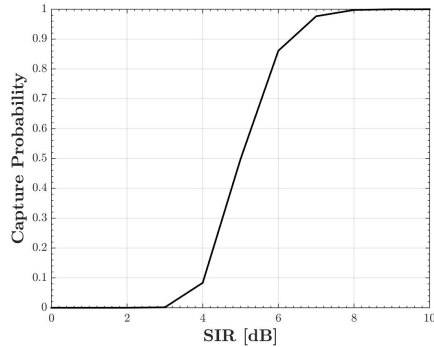

Fig. 19: Capture probability versus SIR: $n=10$, pksize $=512$ bytes, $\mathrm{CBR}=12 \mathrm{Mbps}, 5 \mathrm{X} 0$ config.

ing the duty cycle period $T_{d c}$ so that the collision probability between the two systems will reduce according to equation (9). Figure 18 confirms what said previously.

Finally, we investigate the capture effect included in our model which is somewhat another solution to reduce the negative impact on Wi-Fi. Our model can determine the relation between the capture capability and the interference caused by LTE-U as shown in Figure 19. The Signal-toInterference Ratio (SIR) is varied from $0 \mathrm{~dB}$ to $10 \mathrm{~dB}$. The capture probability shown in the Figure is computed through equation (27), combined with the adequate BER model of OFDM-based WLAN [18]. The plot determines exactly the SIR lower bound before which all collisions will cause a frame loss (no capture effect), and the SIR upper bound beyond which LTE-U and Wi-Fi can coexist together without any impact (Wi-Fi alone). The Wi-Fi throughput and collision probability are also computed in Tables II and III. Likewise, when $S I R \simeq 0 \mathrm{~dB}$, we re-find the results without capture effect, whereas when $\mathrm{SIR}=10 \mathrm{~dB}$, we re-find the results of Wi-Fi alone.

\section{CONCLUSIONS}

In this paper, we have presented two complementary analytical models that provide significant insights for the Wi-Fi performance coexisting with Duty Cycled LTE. Each model represents a different way of describing the LTE-U/Wi-Fi interaction and analyzes a particular aspect about it. These models constitute a general framework relying on random walk theory that can be used to study other LTE-U/Wi-Fi coexistence scenarios. We succeeded to tie up analytically between Duty Cycled LTE configuration parameters and WiFi performance to understand fully how LTE-U can coexists adequately with Wi-Fi networks.

Accordingly, we present several solutions based on tuning the above mentioned parameters including Physical Layer parameters. We examined our models through extensive sim\begin{tabular}{|r|l|l|l|l|l|l|l|l|l|l|l|}
\hline SIR [dB] & 0 & 1 & 2 & 3 & 4 & 5 & 6 & 7 & 8 & 9 & 10 \\
\hline Analytical & 0.415 & 0.4157 & 0.415 & 0.415 & 0.413 & 0.4 & 0.39 & 0.386 & 0.386 & 0.3861 & 0.386 \\
\hline
\end{tabular}

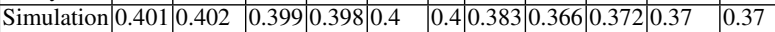

TABLE III: Slot-by-Slot model. Collision probability versus SIR: $\mathrm{n}=10$, pksize $=512$ bytes, $\mathrm{CBR}=12 \mathrm{Mbps}$ and $5 \mathrm{X} 0$ config.

ulations by implementing the Duty Cycles of LTE-U in the NS3 simulator. We prove several facts such as the inability of approximating Duty Cycled LTE by the exponential distribution and the ability of Duty Cycled LTE to be Wi-Fi-Friendly if well configured.

\section{REFERENCES}

[1] Cisco, "Cisco visual networking index: Global mobile data traffic forecast update 2014-2019," 2015.

[2] 3GPP TR 36.889, "3rd generation partnership project; technical specification group radio access network; study on licensed-assisted access to unlicensed spectrum," pp. V13.0.0 (2015-6), Release 13.

[3] E. Almeida, A. M. Cavalcante, R. C. Paiva, F. d. S. Chaves, F. Abinader, R. D. Vieira, S. Choudhury, E. Tuomaala, and K. Doppler, "Enabling lte/wifi coexistence by lte blank subframe allocation," in Communications (ICC), 2013 IEEE International Conference on. IEEE, 2013, pp. 5083-5088

[4] Z. Guan and T. Melodia, "Cu-lte: Spectrally-efficient and fair coexistence between lte and wi-fi in unlicensed bands," in IEEE INFOCOM 2016 - The 35th Annual IEEE International Conference on Computer Communications, April 2016, pp. 1-9.

[5] W. J. Hillery, N. Mangalvedhe, R. Bartlett, Z. Huang, and I. Z. Kovacs, "A network performance study of lte in unlicensed spectrum," in 2015 IEEE Globecom Workshops (GC Wkshps). IEEE, 2015, pp. 1-7.

[6] A. Babaei, J. Andreoli-Fang, and B. Hamzeh, "On the impact of 1te-u on wi-fi performance," in 2014 IEEE 25th Annual International Symposium on Personal, Indoor, and Mobile Radio Communication (PIMRC), Sept 2014, pp. 1621-1625.

[7] "Lte in unlicensed spectrum: Harmonious coexistence with wi-fi," White Paper, Qualcomm, 2014. [Online]. Available: https://www.qualcomm.com/media/documents/files/lte-unlicensedcoexistence-whitepaper.pdf

[8] C. Chen, R. Ratasuk, and A. Ghosh, "Downlink performance analysis of lte and wifi coexistence in unlicensed bands with a simple listen-beforetalk scheme," in Vehicular Technology Conference (VTC Spring), 2015 IEEE 81st. IEEE, 2015, pp. 1-5.

[9] R. Zhang, M. Wang, L. X. Cai, X. Shen, L.-L. Xie, and Y. Cheng, "Modeling and analysis of mac protocol for lte-u co-existing with wifi," in 2015 IEEE GLOBECOM. IEEE, 2015, pp. 1-6.

[10] A. M. Voicu, L. Simiæ, and M. Petrova, "Coexistence of pico- and femto-cellular lte-unlicensed with legacy indoor wi-fi deployments," in 2015 IEEE ICC Workshop, June 2015, pp. 2294-2300.

[11] H. Wu, Y. Peng, K. Long, S. Cheng, and J. Ma, "Performance of reliable transport protocol over ieee 802.11 wireless lan: analysis and enhancement," in INFOCOM 2002. Proceedings. IEEE, vol. 2. IEEE, 2002, pp. 599-607.

[12] G. Bianchi, "Performance analysis of the ieee 802.11 distributed coordination function," Selected Areas in Communications, IEEE Journal on, vol. 18 , no. 3 , pp. 535-547, 2000.

[13] I. Tinnirello, G. Bianchi, and Y. Xiao, "Refinements on ieee 802.11 distributed coordination function modeling approaches," Vehicular Technology, IEEE Transactions on, vol. 59, no. 3, pp. 1055-1067, 2010.

[14] I. C. S. L. M. S. Committee et al., "Ieee std 802.11. wireless lan medium access control (mac) and physical layer (phy) specifications," 1997.

[15] S. M. Ross, Introduction to probability models. Academic press, 2014.

[16] J. Sheng and K. S. Vastola, "Performance modeling of 802.11 ad hoc networks with time-varying carrier sense range and physical capture capability," in IEEE GLOBECOM. IEEE, 2008, pp. 1-5.

[17] P. Fuxjaeger and S. Ruehrup, "Validation of the ns-3 interference model for ieee802. 11 networks," in 2015 8th IFIP Wireless and Mobile Networking Conference (WMNC). IEEE, 2015, pp. 216-222.

[18] M. Lacage and T. R. Henderson, "Yet another network simulator," in Proceeding from the 2006 workshop on ns-2: the IP network simulator. ACM, 2006, p. 12. 